\title{
Physical Performance Is Associated with Executive Functioning in Older African American Women
}

\author{
Brooke C. Schneider ${ }^{1}$ and Peter A. Lichtenberg ${ }^{2}$ \\ ${ }^{1}$ Psychology Service, VA Greater Los Angeles Healthcare Center, Los Angeles, CA 90073, USA \\ ${ }^{2}$ Institute of Gerontology, Wayne State University, Detroit, Michigan 48202, USA \\ Correspondence should be addressed to Brooke C. Schneider, schneider.brooke@gmail.com
}

Received 1 September 2010; Revised 1 December 2010; Accepted 4 January 2011

Academic Editor: Iris Reuter

Copyright (C) 2011 B. C. Schneider and P. A. Lichtenberg. This is an open access article distributed under the Creative Commons Attribution License, which permits unrestricted use, distribution, and reproduction in any medium, provided the original work is properly cited.

\begin{abstract}
An older adult's ability to perform physical tasks is predictive of disability onset and is associated with declines in cognition. Risk factors for physical performance declines among African Americans, a group with the highest rates of disability, remain understudied. This study sought to identify demographic, health, and cognitive factors associated with lower-extremity physical performance in a sample of 106 African American women ages 56 to 91. After controlling for global cognitive functioning (Mini Mental State Exam), physical performance was associated with executive functioning (Stroop Color/Word), but not visuospatial construction (WASI Block Design) or processing speed (Trail Making Test, Part A). Executive functioning remained associated with physical performance after entry of demographic variables, exercise, depression, disease burden, and body mass index (BMI). Age, and BMI were also significant in this model. Executive functioning, age and BMI are associated with lower-extremity physical performance among older African American women.
\end{abstract}

\section{Introduction}

Impairments in physical and cognitive functioning, as well as onset of chronic diseases, are often feared in later life as such changes lead to dependence in tasks of daily living, depression, and hospitalization. Compared to white elders, African American older adults experience a greater number of years exposed to the negative impacts of chronic disease and functional disability [1] making disability intervention especially important for this minority group. The field of gerontology has identified lower-extremity physical performance measures as key for identification of early changes that may lead to disability in older adults. Lower-extremity measures of physical performance are predictive of several outcomes in later life including declines in activities of daily living (ADLs), hospitalization, risk for death, nursing home placement, and hip fracture [2,3]. Reflecting the observed racial and socioeconomic discrepancies in rates of disability among older adults, African American elders score more poorly on performance-based measures of physical function than both white adults [4] and suburban African Americans [5].

While much evidence exists for the relationship between cognitive functioning and ADL declines [6-8], fewer studies have focused on the associations between physical performance and cognition, specifically executive functioning, in minority populations. Executive functioning encompasses a broad range of cognitive abilities such as the planning, sequencing, and execution of complex goal-directed behaviors characteristic of IADLs (instrumental activities of daily living) $[9,10]$. Amongst cognitive domains such as memory, language, visuospatial ability, and psychomotor speed, executive functioning is deemed as essential to preserved functional status $[11,12]$. Prior studies have found that executive functioning is related to mobility and balance among older adults [13-15]; however, this relationship may be attenuated after accounting for disease burden in African American elders [16]. Additionally, it remains unclear whether performance of physical tasks may involve executive functioning more than other domains of cognition 
and whether screening measures of global cognitive functioning, such as the Mini Mental Status Exam (MMSE), which are commonly used in disability risk assessments, may adequately predict physical performance declines [14, $17,18]$.

Neuroimaging studies and clinical observation of agerelated cognitive disorders such as Parkinson's disease and vascular dementia, provide evidence that changes in brain structures result in impairments in both cognitive functioning [9] and physical performance [19]. The frontal subcortical region, implicated in tasks of executive functioning, is particularly sensitive to effects of cardiovascular risk factors (CVRFs) such as atherosclerosis, hypertension, stroke, and diabetes [20-24]. Vascular burden is associated with presence of white matter hyperintensities (WMHs), brain atrophy and infarcts. Older adults with compromise to the white matter pathways connecting subcortical and frontal regions secondary to vascular processes demonstrate poor executive functioning, slow gait speed and depression [15, 25]. The co-occurrence of these symptoms is associated with greater functional dependency [19], poorer physical performance [15], and mortality [26].

African American adults have higher body mass index (BMI), and a greater number of health conditions, particularly CVRFs such as hypertension and diabetes, when compared to their white, same-gender peers $[27,28]$. Both obesity and inactivity are associated with disability [29]. Exercise interventions have been shown to improve scores on measures of lower-extremity functioning [30] and decrease risk for mortality, frailty, disease [31] and obesity [29]; however, it remains unclear what level of exercise is needed to decrease disability risk. In order to develop interventions and delay disability onset within African American elders, a high-risk group of older adults, further work is needed to understand the impact of medical conditions and the potential benefit of exercise to prevent early disability.

The goals of this study are to (1) identify whether a specific domain of cognition versus global cognitive functioning may be uniquely associated with physical performance; and (2) to examine relationships between physical performance and a range of factors associated with disability risk including cognition, depression, exercise, disease burden, BMI and demographics in a sample of community-dwelling African American older women. Based on previous research it was predicted that executive functioning would be significantly associated with physical performance while other domains of cognition would not and that physical performance would be related to demographics, depression, exercise, health, and cognition.

\section{Methods}

2.1. Sample. Participants were drawn from the Health, Disability and Cognitive Function in Urban African American Older Adults dataset, which includes 130 communitydwelling African American adults between the ages of 55 and 100 who resided in the city of Detroit. This project received approval from the Institutional Review Board, Human Investigation Committee of Wayne State University.
Prior to participation, all participants provided signed consent. Subjects were recruited from independent living centers, community centers and senior apartments through presentations within the community given by the PI. Fliers stating that the aim of the study is to understand "health and cognitive functioning in older African American adults" were also given to potential participants. Individuals were excluded if they (1) did not self-report as African American or black; (2) were unable to speak English fluently; (3) had major hearing or vision loss; and/or (4) were below age 55. Because the dataset contained a significantly greater proportion of females $(87.7 \%)$, male participants were excluded from these analyses.

A summary of participant characteristics is presented in Table 1 and mean raw scores on cognitive measures for the final sample are presented in Table 2. The final sample was comprised of 106 African American women ages 56 to 91 $($ mean $=71.83 ; \mathrm{SD}=7.73)$, and with 6 to 18 years of formal education $($ mean $=12.74 ; \mathrm{SD}=2.45)$.

2.2. Measures and Procedures. Measures reported in this study were administered as part of a larger evaluation that involved data collection on demographics, physical health, cognition, health behaviors, and mental health in urban African American elders for a dissertation project. Data was collected in an individual interview session format by three trained interviewers who were supervised by a research psychologist (PL). Participants were informed of the length of the test battery prior to participation. However, due to participant time restraints leading them to leave early or arrive late, as well as slowness in completing the measures, the battery occasionally had to be shortened or terminated before all measures were completed. This led to slight differences in sample size across measures. The average time to complete the battery was two hours.

2.3. Cognitive Measures. Fuld object memory evaluation (FOME) [32]. The FOME is a measure of verbal memory that involves recall of 10 common objects. Recall trials are separated by a distraction task to minimize the effects of short-term memory.

Mini Mental Status Exam (MMSE) [33]. The MMSE is an 11-item screening tool used to obtain an estimate of an individual's global cognitive functioning and orientation to date, time, and place. Scores range from 0 to 30 with higher scores indicating better cognitive functioning.

Stroop Color/Word subtest (Stroop C/W) [34]. The Stroop C/W test is a measure of processing speed and mental flexibility. The Stroop test is comprised of three subtasks: color word naming, color naming, and naming the color of ink a color name is printed in. For example, saying "green" when the word "red" is printed in green ink. Third subtask is an interference trial that requires inhibition and mental flexibility. Time to completion was recorded for each trial.

Trail Making Test, Part A (TMT-A) [35]. Part A of the TMT is a measure of attention and psychomotor processing speed in which participants are asked to connect numbers in numerical order (1-2-3 and so on) as quickly as possible. Scores are based on the time to completion. 
TABLE 1: Demographic, exercise, BMI and mood characteristics of sample.

\begin{tabular}{lccc}
\hline (Overall $N=106)$ & Mean & SD & Range \\
\hline Age & 71.83 & 7.73 & $56-91$ \\
Education & 12.74 & 2.45 & $6-18$ \\
GDS-15 & 1.78 & 1.89 & $0-9$ \\
Exercise & 2.75 & 2.61 & $0-7$ \\
BMI & 31.39 & 7.91 & $15.7-56.7$ \\
\hline
\end{tabular}

Note: GDS: Geriatric Depression Scale-15 item; BMI: body mass index.

TABle 2: Descriptive statistics for cognitive measures.

\begin{tabular}{lccc}
\hline Measure & Mean & SD & Range \\
\hline FOME & 39.84 & 5.31 & $24-50$ \\
MMSE & 27.05 & 2.18 & $19-30$ \\
Stroop Color/Word & 25.15 & 9.63 & $2-49$ \\
TMT-A & 56.21 & 27.02 & $20-136$ \\
WASI BD & 16.05 & 10.35 & $2-43$ \\
\hline
\end{tabular}

Note: FOME: Fuld Object Memory Evaluation; MMSE: Mini Mental Status Exam; TMT-A: Trail Making Test, Part A; WASI BD: Wechsler Abbreviated Scale of Intelligence, Block Design subtest.

Wechsler Abbreviated Scale of Intelligence (WASI), Block Design subtest [36]. The WASI Block Design subtest is a timed measure of visuoconstructional abilities in which participants use blocks to construct three-dimensional figures from a two-dimensional drawing in the stimulus book.

2.4. Geriatric Depression Scale-15 Item (GDS) [37]. The GDS-15 is a shortened version of the original 30-item screening questionnaire that is presented verbally to the participant. Respondents answer yes or no to questions regarding how they have felt over the last two weeks. Items for which a respondent indicates pathology are given a score of 1 . Total scores range from 0 to 15 , with higher scores indicating greater depressive symptomology.

2.5. Exercise. Participation in exercise was obtained from self-reported answers to the following questions: (1) "Do you participate in a regular program of exercise?" and if yes, then "How many days per week". Participants were asked to provide an estimated number of days between 0 and 7 .

2.6. Health. Participants were asked whether a doctor had ever told them that they have health conditions that were grouped into two disease categories: (1) cardiovascular (i.e., hypertension, stroke, myocardial infarct, congestive heart failure, vascular disease, and diabetes), or (2) general health (i.e., arthritis, chronic obstructive pulmonary disease, gastrointenstinal conditions, kidney disease, and liver disease). Table 3 shows the number of participants that reported each of these health conditions. Participants were also asked to report their estimated current height and weight.

2.7. Short Physical Performance Battery (SPPB). The SPPB used in this study was replicated from methodology used
TABLE 3: Frequencies of health conditions.

\begin{tabular}{lcc}
\hline (Overall $N=106)$ & Frequencies & Percentage \\
\hline Hypertension & 71 & $67 \%$ \\
Myocardial infarct & 11 & $10 \%$ \\
Peripheral vascular disease & 8 & $7 \%$ \\
Diabetes & 23 & $22 \%$ \\
Stroke & 11 & $10 \%$ \\
Congestive heart failure & 11 & $10 \%$ \\
Arthritis & 76 & $72 \%$ \\
Gastrointestinal disease & 25 & $24 \%$ \\
Kidney disease & 7 & $7 \%$ \\
Liver disease & 1 & $.9 \%$ \\
COPD or emphysema & 9 & $8 \%$ \\
\hline
\end{tabular}

in the Established Populations for Epidemiologic Studies of the Elderly (EPESE) studies that examined physical functioning in over 5,000 mostly white older adults [38]. This methodology is described in detail elsewhere $[3,38]$. Lowerextremity function was assessed through the performance of three tasks: standing balance, walking, and chair stands.

Balance was assessed by recording the amount of time each participant could maintain each of the following three poses: semitandem (heel of one foot to the side of the first toe of the other foot), tandem (heel to toe), and side-by-side. Timing stopped when the participant lost balance, grasped for the examiner, or ten seconds had elapsed. According to the Guralnik et al.'s criteria [38], participants received a score of a 1 if they were able to hold a side-by-side position for 10 seconds, but were unable to hold a semi-tandem position; a score of a 2 if they could hold a semi-tandem position for 10 seconds but were unable to hold a full tandem for more than 2 seconds; a score of a 3 if they could stand in full tandem for 3 to 9 seconds; and a score of a 4 if they could stand in full tandem for 10 seconds.

Gait speed was assessed by two 3-meter walks, at a normal everyday pace, which was marked out for each subject in advance. The faster of their two walks was used as their final score which was recorded in quartiles such that a score of $1=\geq 5.6$ seconds; a score of $2=4.4-5.5$ seconds; a score of $3=3.8-4.3$ seconds; and a score of $4=\leq 3.7$.

The final task, chair stands, required the participants to fold their arms across their chest and to stand up from a sitting position once. Upon successful completion of this task, participants were asked to stand up and sit down with their arms across their chest five times as quickly as they could. Times were then recorded into quartiles such that a score of a $1=\geq 16.1$ seconds; a score of a $2=12.9$ to 16.0 seconds; a score of a $3=9.9$ to 12.8 seconds; and a score of a $4=\leq 9.8$ seconds.

Summing the scores for each subtask (standing balance, gait speed, and chair rises) creates a summary performance score that was used in the analyses.

2.8. Statistical Methods. All statistical analyses were performed using PASW Statistics 18 (SPSS Inc., 2009). Participants who were missing data on variables of interest $(n=6)$ 
were excluded from the analyses. All variables were examined to ensure they met assumptions of normality. All variables except BMI were within acceptable ranges; a logarithmic transformation was performed on BMI. The transformed variable was used in all analyses. To initially ascertain the relationships between the SPPB and predictor variables, Pearson product moment correlations were obtained. To examine the relationship between physical performance and specific domains of cognition, a multiple regression analysis was conducted in which SPPB total score was regressed on age, education, and MMSE. Each individual cognitive test (Stroop C/W, TMT-A, MMSE and WASI Block Design) was entered into separate regression analyses. Raw scores were used for all cognitive measures of interest. Next, to examine the relationship between physical performance and demographic variables (i.e., age, and education), cognition, exercise, mood, disease burden and BMI, a hierarchical regression analysis was conducted. In Block 1, SPPB total score was regressed on age and education. To examine the incremental variance accounted for by other variables of interest, Stroop C/W raw score was entered in Block 2, exercise was entered in Block 3, GDS total score was entered in Block 4, total number of both vascular health and general health conditions were entered in Block 5, and BMI was entered in Block 6. For both sets of analyses, a $P$ value of less than .05 was considered significant.

\section{Results}

Examination of preliminary analyses revealed significant bivariate relationships between the SPPB and age $(r=-.34$; $P<.00)$, education $(r=.20 ; P<.04)$, BMI $(r=-.27$; $P<.00)$, exercise $(r=.20 ; P<.05)$, vascular health $(r=-.29 ; P<.00)$, general health $(r=-.25 ; P<.01)$, and the GDS $(r=-.26, P<.01)$. All cognitive variables were also significantly correlated with SPPB performance (MMSE, $r=$ .29, $P<.00$; WASI BD, $r=.24, P<.01$; TMT-A, $r=-.33$, $P<.00$; Stroop C/W, $r=.36, P<.00)$, except the FOME $(r=.18, P=.07)$.

To examine our first hypothesis, a multiple regression was conducted to determine the association of each cognitive domain (i.e., attention, visuospatial skills, and executive functioning) with physical performance after accounting for age, education, and general cognitive functioning (MMSE). In Block 1 , age $(P<.00)$ and MMSE $(P<.05)$ significantly contributed to SPPB scores. Upon entry of each cognitive measure individually in Block 2, only Stroop C/W was significantly associated with physical performance $(P<.05)$. MMSE was nonsignificant with the entry of Stroop C/W. This model accounted for $18.8 \%$ of the variance in physical performance scores; see Table 4 for results.

Next, a hierarchical regression was conducted to ascertain the amount of variance in physical performance accounted for by demographics, cognition, exercise, depression, vascular health, general health and BMI. Based on results of the initial analyses, only Stroop C/W was used to represent cognition in this model. $R^{2}$ change was significant at Block 1 with entry of demographics, $R^{2}=.15, F(2,103)=9.39$, $P<.00$. Age $(P<.00)$ and education $(P<.05)$ were both
TABLe 4: Association of cognition with the SPPB.

\begin{tabular}{lccccc}
\hline Variable & Beta & SE Beta & $\beta$ & Sig. & $\Delta R^{2}$ \\
\hline Stroop C/W & .07 & .03 & .21 & .04 & .03 \\
WASI BD & .03 & .03 & .10 & .32 & .01 \\
TMT-A & -.02 & .01 & -.17 & .09 & .09 \\
\hline
\end{tabular}

Note: Results are based on separate hierarchical regression models for each cognitive test. Block 2 adjusted for age, education and MMSE score. WASI BD: Wechsler Abbreviated Scale of Intelligence, Block Design subtest; TMTA: Trail Making Test, Part A.

significant predictors in Block 1. In Block 2, Stroop C/W significantly improved prediction of SPPB scores $(P<.00)$; $\Delta R^{2}=.05, F(1,102)=7.01, P<.00$. With the addition of Stroop C/W, education became nonsignificant. In Block 3, exercise did not significantly improve prediction $(P>.05)$; $\Delta R^{2}=.02, F(1,101)=2.74, P=.10$. GDS significantly improved prediction $(P<.05)$ in Block $4 ; \Delta R^{2}=.04$, $F(1,100)=5.36, P<.05$. In Block 5 , both vascular health $(P<.05)$ and general health were significant $(P<.05)$; $\Delta R^{2}=.08, F(2,98)=5.93, P<.00$. With the entry of the health variables in Block 5, the GDS became non-significant $(P=.21)$. In Block 6, BMI was significant $(P<.05)$; however, vascular and general health became non-significant $(P>$ $.05) ; \Delta R^{2}=.03, F(1,97)=4.84, P<.03$. In the final model, age, Stroop C/W, and BMI were significant contributors, and accounted for $32.8 \%$ (Adj. $R^{2}$ ) of the variance in SPPB scores. Block 6 results are reported in Table 5.

\section{Discussion}

Confirming our hypothesis, among cognitive domains of memory, attention, and visuospatial construction, only executive functioning was significantly associated with physical performance after controlling for general cognitive functioning. A secondary analysis demonstrated that among a range of factors shown to contribute to disability onset, age, executive functioning, and BMI were associated with physical performance scores. These findings have clinical implications for improving gerontology's understanding of disability and helping clinicians and researchers to design and implement interventions aimed at delaying disability onset among African American women.

Foremost, this study provides further evidence that executive functioning contributes not only to ADL disability onset, but also to declines in physical performance, an early indicator of disability, in African American older adults $[1,17,39]$. Executive functioning accounted for a small, yet significant, proportion of variance in physical performance, and our findings suggest that performance of basic physical tasks included in the SPPB involve executive processes such as inhibition and mental flexibility. An older adult's ability to inhibit attention to extraneous information in the environment and to make appropriate responses is involved in the successful performance of physical tasks. Executive functions may become even more important in complex scenarios older adults face in daily life, such as when avoiding obstacles in their home or when attempting to multitask (i.e., walking and talking). 
TABLE 5: Contribution of demographics, cognition, depression, exercise and health variables to SPPB.

\begin{tabular}{lccccc}
\hline Block 6 & Beta & Std. Error & $\beta$ & $t$ & Sig. \\
\hline Age & -.16 & .04 & -.32 & -3.80 & .00 \\
Education & .09 & .12 & .07 & .76 & .45 \\
Stroop C/W & .06 & .03 & .19 & 2.06 & .04 \\
Exercise & .10 & .11 & .09 & .97 & .33 \\
GDS & -.20 & .15 & -.12 & -1.33 & .19 \\
General health & -.49 & .30 & -.15 & -1.63 & .11 \\
Vascular health & -.46 & .26 & -.15 & -1.75 & .08 \\
BMI & -6.24 & 2.83 & -.20 & -2.20 & .03 \\
\hline
\end{tabular}

Note: BMI: Body Mass Index; GDS: Geriatric Depression Scale.

These findings are supported by and expand upon our previous work using a separate dataset of African American elders from the Detroit area [16]. In this study, two of three measures of executive functioning (Trail Making Test, Part B and Animal Naming) were significantly associated with SPPB scores after controlling for general cognitive functioning. The current study demonstrates that even after examination of four other domains of cognition, only executive functioning is associated with physical performance. Providing convergent evidence of this relationship in a similar study of African American older adults, Nieto and colleagues [13] report that after adjusting for age, gender, comorbidity, global cognition, education levels, and global memory, individuals with poor executive function were four times more likely to have impaired lower-extremity functioning. These results are congruent with several other studies reporting relationships between executive functioning and physical performance in samples of predominantly white older adults $[14,15,40]$. Executive functioning measures such as the Stroop are brief, easy to administer and are well tolerated by older adults. Disability assessment traditionally includes some measure of general cognition, often the MMSE. However, we argue that the addition of executive function measures would improve assessment of disability risk.

Our second analysis provides further information about mechanisms potentially underlying the relationship between physical performance and executive functioning. Along with executive functioning, age and BMI were significantly associated with physical performance while mood and exercise were not. Although both vascular and general health conditions were significantly associated with physical performance, they became non-significant after entry of BMI. BMI is a well-established risk factor for disability in older adults [41]. Outcomes of and contributors to BMI and obesity are difficult to separate; however, obesity is highly associated with medical burden, particularly cardiovascular conditions and arthritis, as well as frailty and decreased exercise tolerance and mobility [42]. Reciprocal influences of several processes lead to greater BMI and increase risk for disability. Associations between vascular burden, obesity, and both physical and cognitive declines is of particular interest [43-45].

Our results support the idea that vascular disease in later life increases an older adult's risk for physical declines, and that physical performance is associated with executive dysfunction. Brain insult to shared neuroanatomical pathways secondary to CVRFs may partially explain mutual declines in cognition and physical performance. Supporting this hypothesis, Leung and colleagues [46] found that Stroop performance was related to activation in the anterior cingulate gyrus, as well as inferior and middle frontal regions; areas implicated in physical performance tasks and sensitive to the impact of vascular burden. Because the frontal lobe integrates informational input from multiple regions of the brain, WMHs and atrophy to shared pathways in the frontal region could result in both physical performance declines and executive dysfunction.

Exercise was not associated with physical performance in our study. Previous work has demonstrated that exercise interventions yield better performance on the SPPB [31, 47] when participants were engaged in moderate exercise for approximately 150 minutes per week. Our ability to find a relationship between exercise and the SPPB may have been attenuated by the amount and intensity of exercise of our participants. Also, physical activity is difficult to accurately measure via self-report as older adults often participate in unstructured, low-intensity physical activities that can be difficult to recall [48]. One final consideration is that exercise is a health behavior, while the variables that were significant in the model, namely BMI and cognition, are the outcome of cumulative lifelong processes. BMI in particular likely reflects lifelong health behaviors, including exercise.

Though depression was not significantly related to physical performance in the final model, it was a significant predictor prior to the entry of vascular and general health, and is related to poorer physical performance in other studies [15]. As such, disease burden may mediate the relationship between depression and physical performance [49]. The relationship between physical performance and depression within samples of African American elders requires further clarification.

There are several limitations to this study. Foremost, due to our small sample size $(N=106)$, our findings should be considered exploratory in nature. Further work is needed to extend and support these findings within a larger sample of African American elders drawn from various geographic regions. Secondly, only single measures were used to represent each cognitive domain. Because the Stroop involves processing speed, as do SPPB tasks, it may be questioned whether the processing speed factor accounts 
for its association with the SPPB. Though three of the five cognitive measures were timed (i.e., Stroop C/W, TMT-A and WASI Block Design), and two involved a motor component (i.e., TMT-A and WASI Block Design), none involved lower-extremity physical performance. Only the Stroop $\mathrm{C} / \mathrm{W}$ was significantly associated with physical performance while other measures that involved speeded processing were not, suggesting that the executive functioning components, mental flexibility, and inhibition, account for its association with physical performance rather than processing speed. In the future it would be of interest to use a broader range of executive functioning measures to determine whether a relationship between executive functioning and physical performance would remain, specifically, for untimed measures.

Because the SPPB is designed to measure only lowerextremity function, this may be viewed as a limitation of our study. However, measures of lower, versus upper, extremity performance are more predictive of future disability because lower extremity function has a greater impact on an older adult's ability to remain independent [2]. Additionally, performances on lower-extremity measures decline across time more than performances on upper-extremity measures suggesting that they are a more sensitive indicator of change in functional status [50]. Self-reported disability is also more strongly correlated with lower-extremity than upperextremity tasks [51].

Finally, many studies fail to examine racial differences or race-specific relationships with regard to risk factors for disability. Though not directly compared, the risk factors in this study are similar to those identified in previous studies that used primarily white older adults. Differences in comorbidity account for African American/white disparities in disability rates, suggesting that disease burden may contribute to disability differently in African Americans versus whites. Though there is generally greater variance among individuals in a group than between groups, disease burden may present an exception. African American older adults experience greater comorbidity at an earlier age of onset of chronic diseases, and have a greater average BMI than their white counterparts $[28,52]$. Additionally, many social factors contribute to differences in healthcare seeking and health behaviors in African Americans versus whites. These factors suggest that pathways to disability may operate differently in African Americans.

\section{Conclusions}

Study results provide further information regarding correlates of physical performance, an indicator of predisability, in older African American women. Foremost, they support previous work demonstrating that executive functioning is related to physical performance and may serve as an indicator of disability risk within African American elders. BMI, which is highly associated with disease burden and health behaviors, was also related to physical performance. Disease processes may underlie the relationship between physical performance and executive function and serve as an important target for interventions.

\section{References}

[1] S. Y. Moody-Ayers, K. M. Mehta, K. Lindquist, L. Sands, and K. E. Covinsky, "Black-white disparities in functional decline in older persons: the role of cognitive function," Journals of Gerontology A, vol. 60, no. 7, pp. 933-939, 2005.

[2] J. M. Guralnik, L. Ferrucci, E. M. Simonsick, M. E. Salive, and R. B. Wallace, "Lower-extremity function in persons over the age of 70 years as a predictor of subsequent disability," New England Journal of Medicine, vol. 332, no. 9, pp. 556-561, 1995.

[3] J. M. Guralnik, L. Ferrucci, C. F. Pieper et al., "Lower extremity function and subsequent disability: consistency across studies, predictive models, and value of gait speed alone compared with the short physical performance battery," Journals of Gerontology A, vol. 55, no. 4, pp. M221-M231, 2000.

[4] C. F. Mendes De Leon, L. L. Barnes, J. L. Bienias, K. A. Skarupski, and D. A. Evans, "Racial disparities in disability: recent evidence from self-reported and performance-based disability measures in a population-based study of older adults," Journals of Gerontology B, vol. 60, no. 5, pp. S263S271, 2005.

[5] E. M. Andresen and D. K. Miller, "The future (history) of socioeconomic measurement and implications for improving health outcomes among African Americans," Journals of Gerontology A, vol. 60, no. 10, pp. 1345-1350, 2005.

[6] B. P. Yochim, A. Lequerica, S. E. MacNeill, and P. A. Lichtenberg, "Cognitive initiation and depression as predictors of future instrumental activities of daily living among older medical rehabilitation patients," Journal of Clinical and Experimental Neuropsychology, vol. 30, no. 2, pp. 236-244, 2008.

[7] J. K. Johnson, L. Y. Lui, and K. Yaffe, "Executive function, more than global cognition, predicts functional decline and mortality in elderly women," Journals of Gerontology A, vol. 62, no. 10, pp. 1134-1141, 2007.

[8] M. S. Lewis and L. S. Miller, "Executive control functioning and functional ability in older adults," Clinical Neuropsychologist, vol. 21, no. 2, pp. 274-285, 2007.

[9] R. Au, J. M. Massaro, P. A. Wolf et al., "Association of white matter hyperintensity volume with decreased cognitive functioning: the Framingham Heart study," Archives of Neurology, vol. 63, no. 2, pp. 246-250, 2006.

[10] D. R. Royall, E. C. Lauterbach, J. L. Cummings et al., "Executive control function: a review of its promise and challenges for clinical research-a report from the Committee on Research of the American Neuropsychiatric Association," Journal of Neuropsychiatry and Clinical Neurosciences, vol. 14, no. 4, pp. 377-405, 2002.

[11] D. A. Cahn-Weiner, P. A. Boyle, and P. F. Malloy, "Tests of executive function predict instrumental activities of daily living in community-dwelling older individuals," Applied Neuropsychology, vol. 9, no. 3, pp. 187-191, 2002.

[12] J. E. Carlson, G. V. Ostir, S. A. Black, K. S. Markides, L. Rudkin, and J. S. Goodwin, "Disability in older adults 2: physical activity as prevention," Behavioral Medicine, vol. 24, no. 4, pp. 157-168, 1999.

[13] M. L. Nieto, S. M. Albert, L. A. Morrow, and J. Saxton, "Cognitive status and physical function in older African Americans," Journal of the American Geriatrics Society, vol. 56, no. 11, pp. 2014-2019, 2008.

[14] N. L. Watson, C. Rosano, R. M. Boudreau et al., "Executive function, memory, and gait speed decline in well-functioning older adults," Journals of Gerontology A, vol. 65, no. 10, pp. 1093-1100, 2010. 
[15] I. Hajjar, F. Yang, F. Sorond et al., "A novel aging phenotype of slow gait, impaired executive function, and depressive symptoms: relationship to blood pressure and other cardiovascular risks," Journals of Gerontology A, vol. 64, no. 9, pp. 994-1001, 2009.

[16] B. C. Schneider and P. A. Lichtenberg, "Executive ability and physical performance in urban Black older adults," Archives of Clinical Neuropsychology, vol. 23, no. 5, pp. 593-601, 2008.

[17] K. Duff, J. W. Mold, and M. M. Roberts, "Walking speed and global cognition: results from the OKLAHOMA Study," Neuropsychology, Development, and Cognition B, vol. 15, no. 1, pp. 31-39, 2008.

[18] J. Verghese, C. Wang, R. B. Lipton, R. Holtzer, and X. Xue, "Quantitative gait dysfunction and risk of cognitive decline and dementia," Journal of Neurology, Neurosurgery and Psychiatry, vol. 78, no. 9, pp. 929-935, 2007.

[19] C. Rosano, E. M. Simonsick, T. B. Harris et al., "Association between physical and cognitive function in healthy elderly: the health, aging and body composition study," Neuroepidemiology, vol. 24, no. 1-2, pp. 8-14, 2005.

[20] M. M. B. Breteler, J. C. Van Swieten, M. L. Bots et al., "Cerebral white matter lesions, vascular risk factors, and cognitive function in a population-based study: the Rotterdam study," Neurology, vol. 44, no. 7, pp. 1246-1252, 1994.

[21] C. Dufouil, A. De Kersaint-Gilly, V. Besançon et al., "Longitudinal study of blood pressure and white matter hyperintensities: the EVA MRI cohort," Neurology, vol. 56, no. 7, pp. 921926, 2001.

[22] A. Ylikoski, T. Erkinjuntti, R. Raininko, S. Sarna, R. Sulkava, and R. Tilvis, "White matter hyperintensities on MRI in the neurologically nondiseased elderly: analysis of cohorts of consecutive subjects aged 55 to 85 years living at home," Stroke, vol. 26, no. 7, pp. 1171-1177, 1995.

[23] T. Akisaki, T. Sakurai, T. Takata et al., "Cognitive dysfunction associates with white matter hyperintensities and subcortical atrophy on magnetic resonance imaging of the elderly diabetes mellitus Japanese elderly diabetes intervention trial (J-EDIT)," Diabetes/Metabolism Research and Reviews, vol. 22, no. 5, pp. 376-384, 2006.

[24] B. Ovbiagele and J. L. Saver, "Cerebral white matter hyperintensities on MRI: current concepts and therapeutic implications," Cerebrovascular Diseases, vol. 22, no. 2-3, pp. 83-90, 2006.

[25] B. T. Mast, T. Miles, B. W. Penninx et al., "Vascular disease and future risk of depressive symptomology in older adults: findings from the Health, Aging and Body Composition Study," Biological Psychiatry, vol. 64, no. 4, pp. 320-326, 2008.

[26] L. P. Fried, R. A. Kronmal, A. B. Newman et al., "Risk factors for 5-year mortality in older adults: the cardiovascular health study," Journal of the American Medical Association, vol. 279, no. 8, pp. 585-592, 1998.

[27] R. N. Rooks, E. M. Simonsick, L. M. Klesges, A. B. Newman, H. N. Ayonayon, and T. B. Harris, "Racial disparities in health care access and cardiovascular disease indicators in black and white older adults in the health ABC study," Journal of Aging and Health, vol. 20, no. 6, pp. 599-614, 2008.

[28] R. A. Bulatao and N. B. Anderson, Eds., Understanding Racial and Ethnic Differences in Health in Late Life: A Research Agenda, National Academies Press, Washington, DC, USA, 2004, for the Committee on Population, Division of Behavioral and Social Sciences and Education, National Research Council.

[29] Y. Wu, S. H. McCrone, and H. J. Lai, "Health behaviors and transitions of physical disability among community-dwelling older adults," Research on Aging, vol. 30, no. 5, pp. 572-591, 2008.

[30] R. A. Fielding, J. Katula, M. E. Miller et al., "Activity adherence and physical function in older adults with functional limitations," Medicine and Science in Sports and Exercise, vol. 39, no. 11, pp. 1997-2004, 2007.

[31] M. E. Nelson, W. J. Rejeski, S. N. Blair et al., "Physical activity and public health in older adults: recommendation from the American College of Sports Medicine and the American Heart Association," Medicine and Science in Sports and Exercise, vol. 39, no. 8, pp. 1435-1445, 2007.

[32] P. A. Fuld, Fuld Object-Memory Evaluation, Stoelting, Wood Dale, Ill, USA, 1997.

[33] M. F. Folstein, S. E. Folstein, and P. R. McHugh, "Mini-mental state: a practical method for grading the cognitive state of patients for the clinician," Journal of Psychiatric Research, vol. 12, no. 3, pp. 189-198, 1975.

[34] C. J. Golden, Stroop Color and Word Test, Stoelting, Chicago, Ill, USA, 1978.

[35] R. M. Reitan, "Validity of the trail making test as an indicator of organic brain damage," Perceptual Motor Skills, vol. 8, pp. 271-276, 1958.

[36] D. Wechsler, Wechsler Abbreviated Scale of Intelligence, The Psychological Corporation, San Antonio, Tex, USA, 1999.

[37] J. I. Sheikh and J. A. Yesavage, Clinical Gerontology: A Guide to Assessment and Intervention, The Haworth Press, New York, NY, USA, 1986.

[38] J. M. Guralnik, E. M. Simonsick, L. Ferrucci et al., "A short physical performance battery assessing lower extremity function: association with self-reported disability and prediction of mortality and nursing home admission," Journals of Gerontology, vol. 49, no. 2, pp. M85-M94, 1994.

[39] H. H. Atkinson, C. Rosano, E. M. Simonsick et al., "Cognitive function, gait speed decline, and comorbidities: the health, aging and body composition study," Journals of Gerontology A, vol. 62 , no. 8, pp. 844-850, 2007.

[40] R. Vazzana, S. Bandinelli, F. Lauretani et al., "Trail making test predicts physical impairment and mortality in older persons," Journal of the American Geriatrics Society, vol. 58, no. 4, pp. 719-723, 2010.

[41] C. M. Apovian, C. M. Frey, G. C. Wood, J. Z. Rogers, C. D. Still, and G. L. Jensen, "Body mass index and physical function in older women," Obesity Research, vol. 10, no. 8, pp. 740-747, 2002.

[42] C. M. Apovian, D. T. Villareal, R. F. Kushner, and S. Klein, "Obesity in older adults: technical review and position statement of the American Society for Nutrition and NAASO, The Obesity Society," American Journal of Clinical Nutrition, vol. 82, no. 5, pp. 923-934, 2005.

[43] X. Cui, J. M. Lyness, X. Tu, D. A. King, and E. D. Caine, "Does depression precede or follow executive dysfunction? Outcomes in older primary care patients," American Journal of Psychiatry, vol. 164, no. 8, pp. 1221-1228, 2007.

[44] D. E. Barnes, G. S. Alexopoulos, O. L. Lopez, J. D. Williamson, and K. Yaffe, "Depressive symptoms, vascular disease, and mild cognitive impairment: findings from the cardiovascular health study," Archives of General Psychiatry, vol. 63, no. 3, pp. 273-280, 2006.

[45] R. K. Bhalla, M. A. Butters, J. T. Becker et al., "Patterns of mild cognitive impairment after treatment of depression in the elderly," American Journal of Geriatric Psychiatry, vol. 17, no. 4, pp. 308-316, 2009.

[46] H. C. Leung, P. Skudlarski, J. C. Gatenby, B. S. Peterson, and J. C. Gore, "An event-related functional MRI study of the Stroop 
color word interference task," Cerebral Cortex, vol. 10, no. 6, pp. 552-560, 2000.

[47] J. D. Williamson, M. Espeland, S. B. Kritchevsky et al., "Changes in cognitive function in a randomized trial of physical activity: results of the lifestyle interventions and independence for elders pilot study," Journals of Gerontology A, vol. 64, no. 6, pp. 688-694, 2009.

[48] J. S. Brach, J. M. VanSwearingen, A. B. Newman, and A. M. Kriska, "Identifying early decline of physical function in community-dwelling older women: performance-based and self-report measures," Physical Therapy, vol. 82, no. 4, pp. 320$328,2002$.

[49] B. C. Schneider and P. A. Lichtenberg, "Depression in later life: an analysis of the activity restriction model," in Proceedings of the 116th Annual Convention of the American Psychological Association, Boston, Mass, USA, 2008.

[50] G. Onder, B. W. J. H. Penninx, P. Lapuerta et al., "Change in physical performance over time in older women: the women's health and aging study," Journals of Gerontology A, vol. 57, no. 5, pp. M289-M293, 2002.

[51] E. M. Simonsick, J. D. Kasper, J. M. Guralnik et al., "Severity of upper and lower extremity functional limitation: scale development and validation with self-report and performancebased measures of physical function," Journals of Gerontology B, vol. 56, no. 1, pp. S10-S19, 2001.

[52] D. D. Dunlop, J. Song, L. M. Manheim, M. L. Daviglus, and R. W. Chang, "Racial/ethnic differences in the development of disability among older adults," American Journal of Public Health, vol. 97, no. 12, pp. 2209-2215, 2007. 


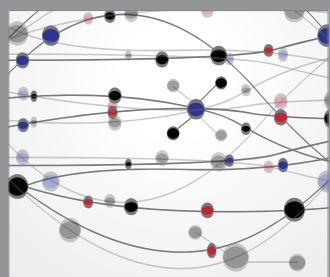

The Scientific World Journal
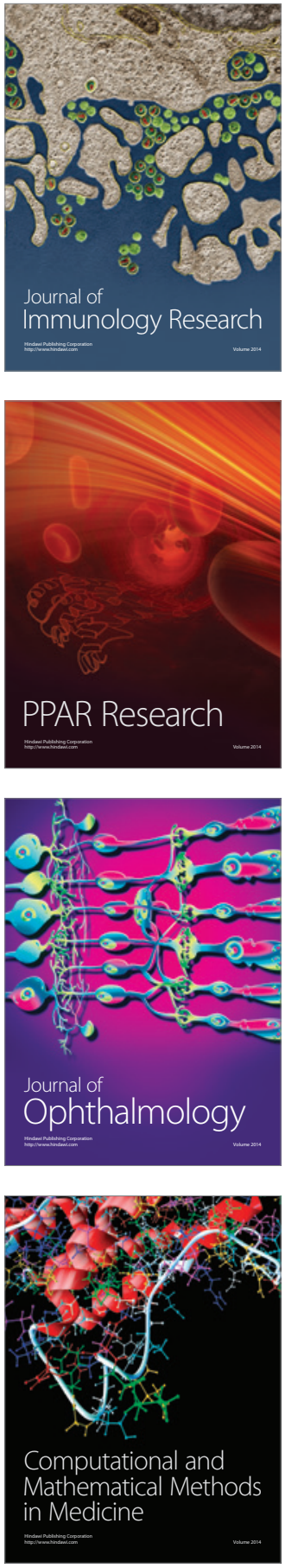

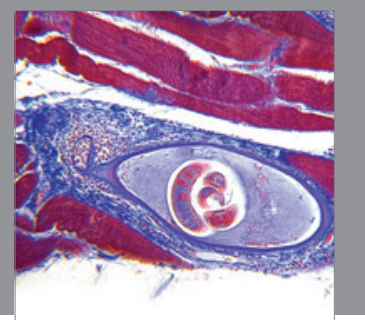

Gastroenterology

Research and Practice
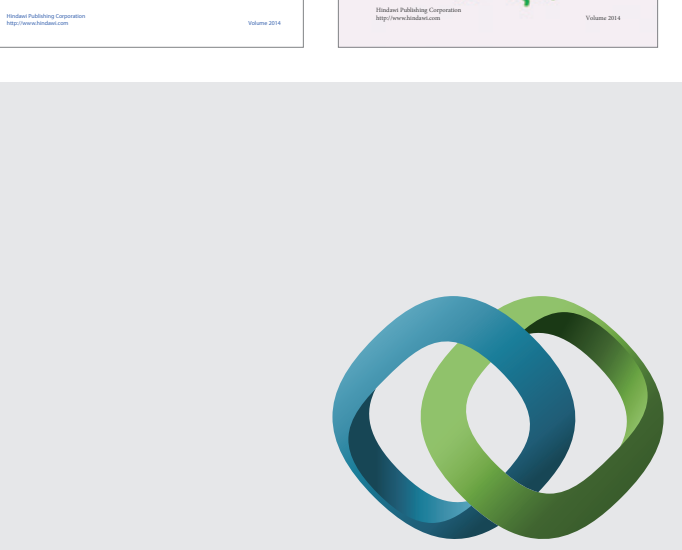

\section{Hindawi}

Submit your manuscripts at

http://www.hindawi.com
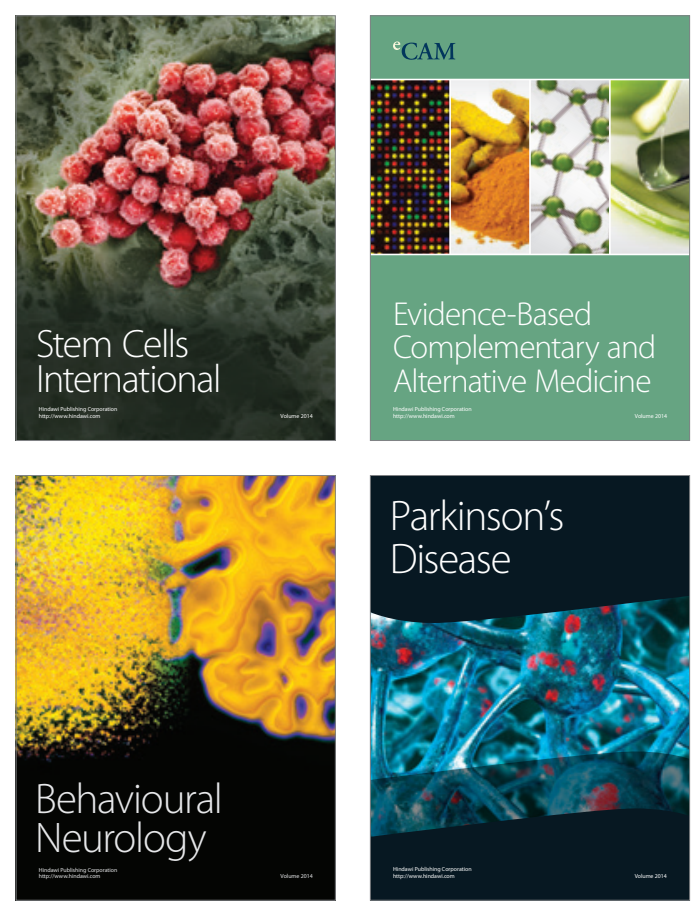

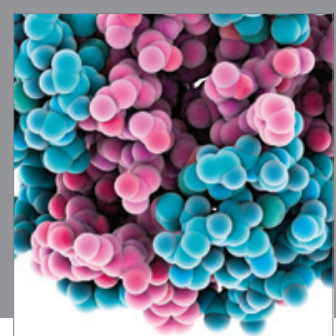

Journal of
Diabetes Research

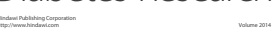

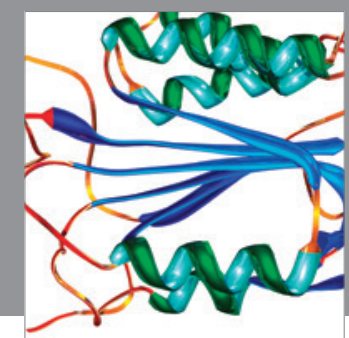

Disease Markers
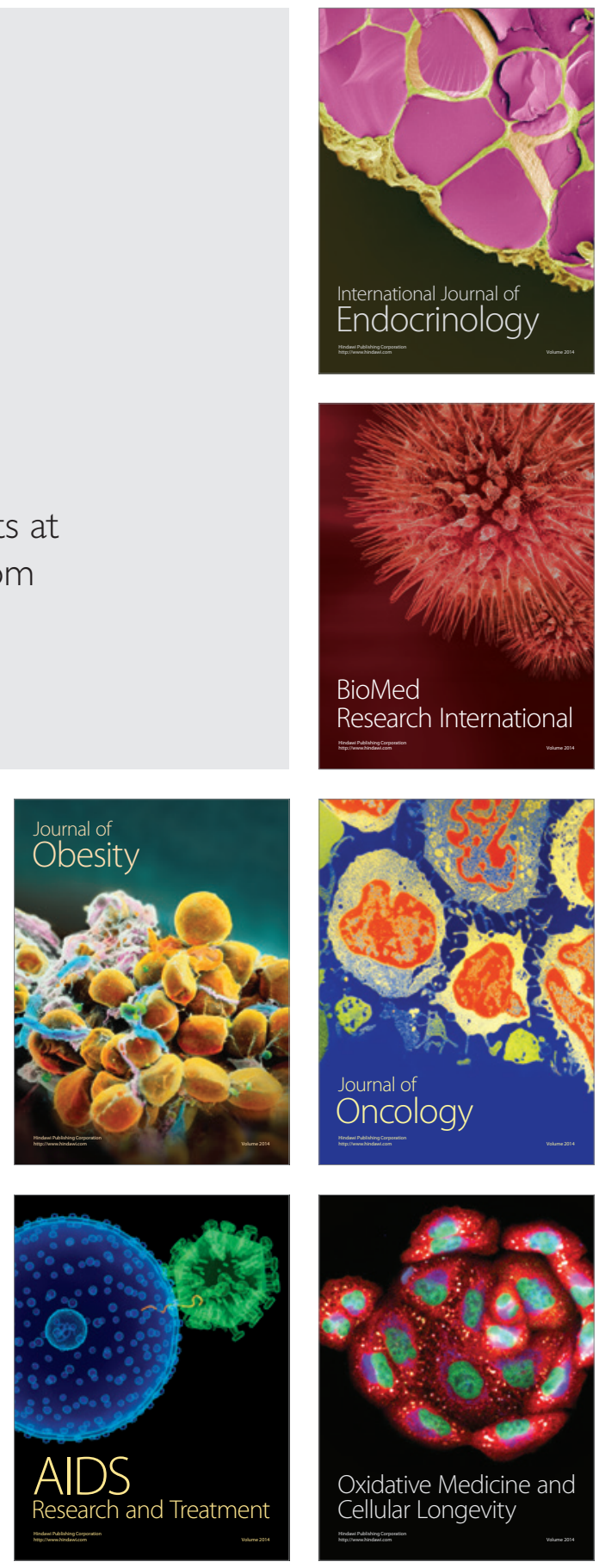